\title{
Avaliação da influência de características sensoriais e do conhecimento nutricional na aceitação do chá-mate
}

\author{
Evaluation of the influence of the sensory characteristics and nutritional \\ knowledge in the acceptance of mate tea
}

\author{
Helohá de Castro Barboza1', Mariana de Melo Cazal ${ }^{1 *}$ \\ ${ }_{1}^{1}$ Faculdade Redentor, Departamento de Nutrição, Itaperuna/RJ - Brasil
}

\section{*Corresponding Author}

Mariana de Melo Cazal, Faculdade Redentor, Departamento de Nutrição, Rodovia BR-356, 25, Cidade Nova, CEP: 28300-000, Itaperuna/RJ - Brasil, e-mail: marianademelocazal@yahoo.com.br

Cite as: Evaluation of the influence of the sensory characteristics and nutritional knowledge in the acceptance of mate tea. Braz. J. Food Technol., v. 21, e2017075, 2018.

Received: May 24, 2017; Accepted: Aug. 29, 2017

\section{Resumo}

No âmbito empresarial, as estratégias de marketing voltadas para assuntos relacionados à nutrição têm sido a principal vantagem competitiva. Diante disso, o objetivo deste trabalho foi avaliar a influência de características sensoriais e do conhecimento nutricional na aceitação do chá-mate, visando identificar o comportamento do consumidor frente a este tipo de informação. Foi utilizado chá-mate tostado, tipo a granel, adquirido no comércio local e preparado de acordo com a recomendação descrita na embalagem. Para o teste afetivo de aceitação, foram recrutados 30 consumidores do produto. O recrutamento ocorreu por meio de um questionário abrangendo questões sobre o perfil dos consumidores, hábitos de consumo e disponibilidade para participar da pesquisa. Foram realizadas avaliações de aceitação sensorial em duas sessões (teste cego e com informação), utilizando-se uma escala hedônica de nove pontos, para avaliar os atributos cor, sabor, aroma e impressão global. Em relação ao perfil dos julgadores, $67 \%$ pertenciam ao sexo feminino, $50 \%$ relataram consumir chá-mate raramente e $37 \%$ afirmaram observar as informações nutricionais no rótulo. Houve diferença significativa para os atributos cor e sabor, entre as sessões cega e com informação. Pode-se concluir que o chá-mate apresentou boa aceitação pelos participantes e que o conhecimento nutricional influenciou positivamente na aceitação da bebida.

Palavras-chave: Chá-mate; Avaliação sensorial; Consumidor; Saúde.

\section{Abstract}

In the business field, marketing strategies focused on nutritional issues have been the main competitive advantage. Therefore the objective of this study was to evaluate the influence of the sensory characteristics and nutritional knowledge on the acceptance of mate tea, in order to identify consumer behaviour in relation to this type of information. Toasted mate tea was used, purchased in bulk at the local shops and prepared according to the manufacturer's instructions, as described on the package. Thirty consumers were recruited for the affective acceptance test. Recruitment was via a questionnaire including questions about the profile of the consumers, consumption habits and willingness to participate in the survey. The sensory acceptance evaluations were carried out in two sessions (blind test and with information) using a nine point hedonic scale to evaluate the attributes of colour, taste, aroma and overall impression. Regarding the profile of the judges, $67 \%$ were female, $50 \%$ reported consuming mate tea rarely and $37 \%$ reported reading the nutritional information on the label. There were significant differences for the attributes of colour and flavour between the blind and informed sessions. It was concluded that the mate tea was well accepted by the participants and that nutritional knowledge positively influenced0 acceptance of the drink.

Keywords: Mate tea; Sensory evaluation; Consumer; Health. 


\section{Introdução}

O chá é o alimento mais consumido no mundo, perdendo apenas para a água. O hábito de consumir chá vem desde a Antiguidade, quando as plantas eram utilizadas como medicamentos para a prevenção, a cura e o tratamento de distúrbios, disfunções ou doenças, em seres humanos e animais (BATISTA et al., 2009; LAMARÃO; FIALHO, 2009; SAIGG; SILVA, 2009). A Erva-Mate, cujo nome científico é /lex paraguariensis, pertence à família das plantas Aquifoliaceaes, de origem subtropical, nativa da América do Sul e é muito consumida na Região Sudeste do Brasil, na forma de chá (chá-mate) (BIASI et al., 2009).

No âmbito empresarial, as estratégias de marketing relacionadas à nutrição têm sido a principal vantagem competitiva, uma vez que os consumidores estão cada vez mais conscientes da direta relação da boa alimentação com a saúde. Diante disso, tem-se aumentado o desenvolvimento de produtos à base de erva-mate, para atender à demanda dos consumidores (CHIESA et al., 2012; PRECl et al., 2011; ZANCHETT et al., 2016).

A principal característica não sensorial que as indústrias alimentícias têm adotado para atender às expectativas do público consumidor é a informação nutricional, contida na embalagem dos alimentos. Esta informação assume um papel de destaque em relação a outros elementos de comunicação de marketing, demonstrando, assim, ser uma ferramenta de relevância no processo de decisão de compra (RIBEIRO et al., 2008).

A informação nutricional atua como um elo na comunicação entre produtores e consumidores. São elementos identificadores que, além da função publicitária, servem principalmente como um meio de informação, o qual, durante o processo de decisão de compra, permite ao consumidor a escolha saudável dos produtos alimentícios, tornando-se um fator determinante na seleção dos alimentos (CAVADA et al., 2012).

Diante disso, considerando-se que o conhecimento nutricional é capaz de facilitar o uso das informações nutricionais, devido à facilidade de interpretar e compreender os benefícios alimentares, este estudo objetivou avaliar a influência de características sensoriais e do conhecimento nutricional na aceitação do chá-mate, visando identificar o comportamento do consumidor frente a este tipo de informação.

\section{Material e métodos}

Inicialmente, o estudo foi submetido e aprovado pelo Comitê de Ética em Pesquisa da Faculdade Redentor (parecer n. ${ }^{\circ}$ 1.640.108/2016).

\subsection{Amostra}

Foi utilizado chá-mate tostado a granel, adquirido no comércio local da cidade de Itaperuna, Rio de Janeiro. A escolha do produto utilizado para o estudo foi em razão de a marca ser líder no mercado nacional. A infusão ocorreu de acordo com a recomendação descrita na embalagem, em que 12,5 g de chá-mate foram diluídos em $1.000 \mathrm{~mL}$ de água fervente, em temperatura acima de $90^{\circ} \mathrm{C}$. Em seguida, o chá foi resfriado e servido à temperatura ambiente.

Ao chá, não foi adicionado açúcar. De acordo com Belitz et al. (2009), a presença do açúcar, mesmo em quantidades pequenas, pode influenciar na percepção sensorial do aroma.

\subsection{Teste sensorial}

Para o teste afetivo de aceitação, foram recrutados 30 consumidores de chá-mate. O questionário de recrutamento abrangeu questões sobre o perfil dos consumidores, hábitos de consumo e disponibilidade para participar da pesquisa. Foram excluídos, do estudo, indivíduos com idade inferior a 18 anos, que apresentavam aversão ao chá-mate, que não tinham disponibilidade de tempo para participar da pesquisa ou que estavam com infecção nasal ou bucal. Todos os julgadores assinaram o Termo de Consentimento Livre e Esclarecido, antes de participarem da pesquisa.

Os testes foram conduzidos no Laboratório de Análise Sensorial da Faculdade Redentor, em duas sessões. Nestas, os julgadores degustaram aproximadamente $40 \mathrm{~mL}$ de chá-mate tostado, servidos em copos plásticos descartáveis, codificados com três dígitos aleatórios.

Na primeira sessão (teste cego), os consumidores degustaram o chá-mate sem receber nenhuma informação prévia do produto. Na segunda sessão (teste com informação), os consumidores, antes da degustação da amostra de chá-mate, receberam um panfleto contendo informações sobre os benefícios do consumo do chá-mate comprovados cientificamente, como efeito antioxidante, anti-inflamatório, termogênico e melhoria do perfil lipídico.

Para avaliação sensorial do chá-mate, foi utilizada uma ficha contendo uma escala hedônica estruturada de nove pontos, em que foi solicitado o julgamento em relação à aceitação do produto, atribuindo notas que variam de 1 "desgostei extremamente" à nota 9 "gostei extremamente" (DELLA LUCIA et al., 2010). A aceitação sensorial foi avaliada quanto aos atributos cor, odor, sabor e impressão global, nas duas sessões.

As análises foram realizadas em cabines individuais com incidência de luz branca, sob condições controladas. Em cada cabine, havia um copo com água, para que o julgador pudesse neutralizar as sensações do sabor da amostra anterior.

\subsection{Análise estatística}

A comparação dos resultados da análise sensorial entre o teste cego e o teste com informação foi realizada pelo teste t para amostras pareadas, considerando-se 
um nível de $5 \%$ de probabilidade. As análises estatísticas foram conduzidas no software SigmaPlot versão 12.0.

\section{Resultados e discussão}

\subsection{Perfil dos julgadores}

Dos 30 julgadores selecionados para compor a equipe sensorial, 67\% $(n=20)$ pertenciam ao sexo feminino e $33 \%(n=10)$ pertenciam ao sexo masculino. Todos os participantes eram estudantes de graduação e apresentavam idade entre 19 e 36 anos, com idade média de 23 anos.

De acordo com Cobra (2009), as mulheres representam $60 \%$ da população americana que mais consome chá. Elas se destacam entre os homens, pois estão cada vez mais preocupadas com a saúde e a aparência física, e em busca de uma vida mais saudável, características que estão relacionadas com o consumo de produtos naturais.

Os hábitos de consumo dos julgadores estão apresentados na Tabela 1. Metade dos participantes reportou consumir chá-mate raramente, enquanto que

Tabela 1. Hábitos de consumo dos 30 julgadores participantes do estudo.

\begin{tabular}{|c|c|}
\hline Questões & $\mathbf{N}(\%)$ \\
\hline \multicolumn{2}{|l|}{ Consome chá-mate? } \\
\hline Não & $0(0)$ \\
\hline $\operatorname{sim}$ & $30(100)$ \\
\hline \multicolumn{2}{|l|}{ Com que frequência? } \\
\hline Diariamente & $2(6,67)$ \\
\hline Semanalmente & $5(16,67)$ \\
\hline Mensalmente & $8(26,67)$ \\
\hline Raramente & $15(50)$ \\
\hline \multicolumn{2}{|c|}{ Além do chá-mate, qual outro chá que você consome? } \\
\hline Erva-doce & $2(6,67)$ \\
\hline Chá preto & $2(6,67)$ \\
\hline Frutas & $2(6,67)$ \\
\hline Chá de camomila & $10(33,33)$ \\
\hline Chá verde & $11(36,67)$ \\
\hline Outros & $3(10)$ \\
\hline \multicolumn{2}{|c|}{$\begin{array}{l}\text { Com que frequência você observa os rótulos dos alimentos } \\
\text { e bebidas? }\end{array}$} \\
\hline Nunca & $4(13,33)$ \\
\hline Frequentemente & $7(23,33)$ \\
\hline Sempre & $8(26,67)$ \\
\hline Raramente & $11(36,67)$ \\
\hline \multicolumn{2}{|c|}{$\begin{array}{l}\text { Quais são as informações que sempre são observadas nas } \\
\text { embalagens dos alimentos e bebidas? }\end{array}$} \\
\hline Informações sobre aditivos & $5(16,67)$ \\
\hline Design & $9(30)$ \\
\hline Informações nutricionais & $11(36,67)$ \\
\hline Marca & $12(40)$ \\
\hline Informações sobre ingredientes & $13(43,33)$ \\
\hline Preço & $19(63,33)$ \\
\hline Prazo de validade & $24(80)$ \\
\hline
\end{tabular}

apenas $6,67 \%(n=2)$ consumiam diariamente. Isso demonstra que a maioria dos julgadores não apresentava o hábito de consumir chá-mate regularmente, o que pode ter cooperado para um viés no estudo.

No que diz respeito à frequência de leitura dos rótulos dos alimentos e bebidas, metade dos consumidores declarou ler os rótulos raramente $(36,67 \%)$ ou nunca $(13,33 \%)$, sendo que os itens mais observados foram o prazo de validade (80\%) e o preço $(63,33 \%)$ (Tabela 1$)$. Em contrapartida, Dantas (2005) observou que a porcentagem das pessoas que declararam possuir o hábito de ler os rótulos das embalagens sempre ou frequentemente (77\%) foi maior do que as que liam às vezes (21\%). Ainda nesse estudo, foi observado também que os consumidores possuíam o hábito de analisar principalmente o prazo de validade (95\%), o preço (89\%) e a marca $(69 \%)$ dos produtos alimentícios, corroborando com os resultados apresentados no presente estudo. Francisco et al. (2014) observaram que 59\% dos participantes do seu estudo declararam ler os rótulos "às vezes" ou "ocasionalmente", sendo o prazo de validade ( $91 \%$ ) e a marca $(75 \%)$, as informações mais observadas pelos consumidores.

É importante destacar que, em todos os trabalhos citados, incluindo o estudo atual, a maioria dos participantes declarou não realizar a leitura completa dos rótulos, atentando-se apenas ao prazo de validade, ao preço e à marca. $\mathrm{O}$ prazo de validade de um alimento ou bebida expressa o prazo, após o qual, o alimento está legalmente impróprio para o consumo. Após o prazo de validade, o produto pode perder suas características, tais como cor, sabor, textura, odor, valor nutricional e, principalmente, pode apresentar deterioração de origem microbiológica e trazer riscos e/ou danos à saúde do consumidor. Dessa forma, considerando-se que o prazo de validade é um fator determinante na qualidade sensorial, nutricional e higienicossanitária do produto alimentício, fica claro que o consumidor prioriza sua saúde e segurança no momento da compra do alimento (MACHADO et al., 2006).

Ressalta-se que 36,67\% ( $n=11)$ dos consumidores afirmaram observar as informações nutricionais no rótulo. Embora a leitura de rótulos para obter informações nutricionais ainda não seja um hábito da maioria dos consumidores, estas informações, quando bem utilizadas e compreendidas pela população, torna-se um potente instrumento de educação nutricional. Por outro lado, a falta de entendimento da linguagem utilizada nos rótulos faz com que as informações disponibilizadas não sejam utilizadas e, consequentemente, não demonstram nenhum impacto na escolha dos alimentos (SILVA, 2003).

Os dados apresentados comprovam a necessidade de fortalecer a leitura das informações presentes no rótulo dos alimentos, uma vez que este é o principal meio de conexão entre o consumidor e o produto. O rótulo é capaz de interferir no processo de decisão de compra, 
contribuir para a escolha de alimentos mais saudáveis e, consequentemente, reduzir a obesidade e as doenças crônicas não transmissíveis, favorecendo a segurança alimentar e nutricional. Assim, é fundamental que a informação nutricional passe a ser um instrumento de notícia que favoreça o conhecimento dos consumidores sobre alimentação e saúde (SOUZA et al., 2011; STEFANO; CASAROTTO FILHO, 2012).

\subsection{Testes de aceitação}

As médias do teste de aceitação do chá-mate para os atributos cor, sabor, odor e impressão global, realizado nas duas sessões, estão apresentadas na Tabela 2. Cada atributo obteve uma média entre os 30 julgadores. Observou-se que houve um aumento das médias das notas de aceitação do chá-mate do teste cego para o teste com informação, para todos os atributos, sendo que, para cor e sabor, houve um aumento significativo nas médias. Antes de receber as informações, as médias de aceitação atribuídas pelos julgadores para os atributos cor e sabor foram 7,4 e 6,6, respectivamente. Após receber a informação dos benefícios para a saúde com o consumo do chá-mate, as médias de aceitação de cor e sabor aumentaram significativamente para 7,9 e 7,2, respectivamente.

Resultados similares foram encontrados por Brazil et al. (2015), os quais, avaliando a aceitação sensorial de damasco em duas sessões, observaram que, antes de receber a informação, a média de aceitação do damasco conferida pelos julgadores foi de 6,31, constatando que, após receber a informação sobre os benefícios para a saúde com o consumo desse alimento, as médias de aceitação aumentaram para 6,64. Esses resultados demonstram que a transmissão do conhecimento nutricional é capaz de melhorar a percepção sensorial do alimento, podendo ser um veículo de educação nutricional apropriado para alterar o comportamento alimentar.

A qualidade do chá é especialmente apreciada por meio de sua aparência (cor, intensidade da cor e turbidez), aroma (floral, doce e "gramado") e sabor (adstringência, amargor e doçura), que são atribuídos à presença de alcaloides, cafeína e catequinas, no chá (BALDUINO, 2012).

Tabela 2. Médias \pm desvio padrão de aceitação da amostra de chá-mate nos testes cego e com informação nutricional $(n=30)$.

\begin{tabular}{lcc} 
& Teste cego & Teste com informação \\
Cor & $7,4 \pm 1,69^{\mathrm{a}}$ & $7,9 \pm 1,67^{\mathrm{b}}$ \\
Odor & $7,3 \pm 1,95^{\mathrm{a}}$ & $7,7 \pm 1,51^{\mathrm{a}}$ \\
Sabor & $6,6 \pm 1,59^{\mathrm{a}}$ & $7,2 \pm 1,55^{\mathrm{b}}$ \\
Impressão global & $7,0 \pm 1,69^{\mathrm{a}}$ & $7,6 \pm 1,67^{\mathrm{a}}$ \\
\hline
\end{tabular}

Médias seguidas de letras iguais na mesma linha indicam não haver diferença significativa no nível de $5 \%$ de probabilidade de erro (Teste t).
De acordo com Teixeira (2009), a cor e a aparência são a apresentação visual, são atributos extrínsecos, que representam o primeiro contato entre o consumidor e o produto, determinando as reações pessoais de aceitação, indiferença ou rejeição. Para Crepaldi (2006), as cores têm a capacidade de despertar sensações, definir comportamentos e provocar reações corporais e psicológicas. Dessa forma, as cores podem ser usadas com finalidade de marketing e propaganda, atendendo às necessidades dos indivíduos, refletindo no processo de compra dos produtos.

Segundo Wang et al. (1999), o sabor é um atributo sensorial exigido para a boa aceitabilidade do produto. O gosto é atribuído aos compostos não voláteis nos alimentos, tais como açúcares, ácidos, sais e limonina. A doçura do chá é conferida pelos aminoácidos, os quais têm o sabor descrito como "umami", e as saponinas, que estão presentes no mate e são responsáveis pelo sabor amargo e pela espuma do chá (BALDUINO, 2012; GNOATTO et al., 2008).

Godoy et al. (2013) observaram que o chá-mate tem grande aceitação pelos consumidores, uma vez que $61 \%$ dos participantes do seu estudo relataram gostar bastante da bebida, principalmente na forma de sachê. Além disso, $61 \%$ dos entrevistados relataram gostar muito de chá adoçado com açúcar. No estudo atual, optou-se por não adoçar o chá-mate, para que o açúcar não interferisse na análise sensorial do produto que estava sendo avaliado, o que pode ter refletido em uma avaliação menos positiva, pois, conforme observado, ainda, por Godoy et al. (2013), a maioria dos consumidores deste chá o prefere adoçado com açúcar.

Com relação aos atributos odor e impressão global, não houve diferença estatisticamente significativa no nível $5 \%$ de probabilidade entre as respostas obtidas nas sessões cega e com informação.

Examinando-se o aumento nas médias das notas de aceitação atribuídas pelo julgador na sessão com informação (Tabela 2), pode-se observar que, de maneira geral, o julgador foi influenciado positivamente pela informação não sensorial apresentada, uma vez que a expectativa gerada pela informação dos benefícios à saúde, na percepção do consumidor, foi conquistada por meio da maior aceitação do chá-mate para os atributos cor e sabor.

Muitas intervenções centradas na educação nutricional têm sido conduzidas com o intuito de aumentar o conhecimento nutricional da população e, consequentemente, melhorar seus hábitos alimentares. Esta medida é importante, uma vez que o entendimento de como os consumidores compreendem um produto saudável colabora de forma relevante para a construção de políticas de educação nutricional (NICASTRO et al., 2008; MARINS et al., 2008). 
Avaliação da influência de características sensoriais e do conhecimento nutricional na aceitação do chá-mate

Barboza, H. C.; Cazal, M. M.

As intervenções nutricionais devem partir da hipótese de que o grau de conhecimento obtido por cada pessoa, ao saber decifrar e identificar as características não sensoriais do produto, principalmente a questão nutricional, poderá modificar as preferências alimentares. A intervenção deve ser aplicada de maneira crítica e reflexiva, voltada para a propagação de conhecimentos e transformação social (ROCHA, 2008; PRATES; SILVA, 2013).

\section{Conclusão}

Os resultados demonstraram que o chá-mate foi bem aceito pelos participantes e o conhecimento nutricional influenciou positivamente na aceitação da bebida, uma vez que houve aumento das médias de aceitação para os atributos cor e sabor, no teste com informação. Dessa forma, é fundamental que a informação nutricional sobre os benefícios do alimento passe a ser um instrumento de notícia que favoreça o conhecimento dos consumidores sobre alimentação e saúde, conduzindo à escolha por produtos mais saudáveis.

\section{Referências}

BALDUINO, M. A. Aplicação da metodologia de engenharia Kansei na análise de consumo de chás. 2012. 90 f. Dissertação (Mestrado em Ciências do Consumo e Nutrição)-Faculdade de Ciências, Universidade do Porto, Porto, 2012.

BATISTA, G. A. P.; CUNHA, C. L. P.; SCARTEZINI, M.; HEYDE, R. V. D.; BITENCOURT, M. G.; MELO, S. F. Estudo prospectivo, duplo cego e cruzado da Camellia sinensis (chá verde) nas dislipidemias. Arquivos Brasileiros de Cardiologia, v. 93, n. 2, p. 128-134, 2009. PMid:19838489. http://dx.doi.org/10.1590/ S0066-782X2009000800010.

BELITZ, H. D.; GROSH, W.; SCHIEBERLE, P. Carbohydrates. In: BELITZ, H. D.; GROSH, W.; SCHIEBERLE, P. Food chemistry. 4th ed. Berlin: Springer, 2009. cap. 4, p. 248-339.

BIASI, B.; GRAZZIOTIN, N. A.; HOFMANN JUNIOR, A. E. Atividade antimicrobiana dos extratos de folhas e ramos da Ilex paraguariensis A. St.-Hil., Aquifoliaceae. Revista Brasileira de Farmacognosia, v. 19, n. 2, p. 582-585, 2009. http://dx. doi. org/10.1590/S0102-695X2009000400013.

BRAZIL, J. M.; LIMA, E. R.; SANTOS, R. A.; VALASQUES JUNIOR, G. L.; MILAGRES, M. P. Nova metodologia de avaliação de resultados em estudos não sensoriais com consumidores no processo de inovação. In: ENCONTRO NACIONAL DE ENGENHARIA DE PRODUÇÃO, 15., 2015, Fortaleza, CE. Anais... Fortaleza: Associação Brasileira de Engenharia de Produção, 2015. p. 1-17.

CAVADA, G. S.; PAIVA, F. F.; HELBIG, E.; BORGES, L. R. Rotulagem nutricional: você sabe o que está comendo? Brazilian Journal of Food Technology, v. 4, n. spe, p. 84-88, 2012. http://dx.doi. org/10.1590/S1981-67232012005000043.
CHIESA, L.; SCHLABITZ, C.; SOUZA, C. F. V. Efeito da adição de erva-mate nas características sensoriais e físico-químicas de barras de cereais. Revista do Instituto Adolfo Lutz, v. 71, n. 1, p. 105-110, 2012

COBRA, M. Administração de marketing no Brasil. 3. ed. Rio de Janeiro: Elsevier, 2009. 560 p.

CREPALDI, L. A influência das cores na decisão de compras: um estudo do comportamento do consumidor no ABC paulista. In: CONGRESSO BRASILEIRO DE CIÊNCIAS DA COMUNICAÇÃO, 29., 2006, Brasília. Anais... Brasília: UnB, 2006. p. 1-14.

DANTAS, N. J. O. Aspectos constitucionais do aleitamento materno: saúde da criança e liberdade econômica. 2005. 230 f. Dissertação (Mestrado em Direito)-Faculdade de Direito, Universidade Metodista de Piracicaba, Piracicaba, 2005.

DELLA LUCIA, S. M.; SOUZA, S.; SARAIVA, S. H.; CARVALHO, R. V.; CARNEIRO, H. C. S. Impacto de características sensoriais e não sensoriais na escolha e na aceitação de iogurte sabor morango. Enciclopédia Biosfera, v. 6, n. 9, p. 1-13, 2010.

FRANCISCO, J. S.; SANTOS, A. F.; BENASSI, M. T. Efeito das informações e características da embalagem na expectativa e aceitação de café solúvel adicionado de café torrado micronizado. Brazilian Journal of Food Technology, v. 17, n. 3, p. 243-251, 2014. http://dx.doi.org/10.1590/1981-6723.1614.

GNOATTO, S. C.; DASSONVILLE-KLIMPT, A.; NASCIMENTO, S.; GALÉRA, P.; BOUMEDIENE, K.; GOSMANN, G.; SONNET, P.; MOSLEMI, S. Evaluation of ursolic acid isolated from Ilex paraguariensis and derivatives on aromatase inhibition. European Journal of Medicinal Chemistry, v. 43, n. 9, p. 1865-1877, 2008. PMid:18192087. http://dx.doi.org/10.1016/j.ejmech.2007.11.021.

GODOY, R. C. B.; DELIZA, R.; GUENO, L. B.; LICODIEDOFF, S.; FRIZON, C. N. T.; RIBANI, R. H.; SANTOS, G. G. Consumer perceptions, attitudes and acceptance of new and traditional mate tea products. Food Research International, v. 53, n. 2, p. 801-807, 2013. http://dx.doi.org/10.1016/j.foodres.2013.02.054.

LAMARÃO, R. C.; FIALHO, E. Aspectos funcionais das catequinas do chá verde no metabolismo celular e sua relação com a redução da gordura corporal. Revista de Nutrição, v. 22, n. 2, p. 257-269, 2009. http://dx.doi.org/10.1590/S1415-52732009000200008.

MACHADO, S. S.; SANTOS, F. O.; ALBINATI, F. L.; SANTOS, L. P. R. Comportamento dos consumidores com relação à leitura de rótulo de produtos alimentícios. Alimentos e Nutrição, v. 17, n. 1, p. 97-103, 2006.

MARINS, B. R.; JACOB, S. C.; PERES, F. Avaliação qualitativa do hábito de leitura e entendimento: recepção das informações de produtos alimentícios. Ciência e Tecnologia de Alimentos, v. 28, n. 3, p. 579-585, 2008. http://dx.doi.org/10.1590/S010120612008000300012.

NICASTRO, H.; DATTILO, M.; SANTOS, T. R.; PADILHA, H. G. V.; ZIMBERG, I. Z.; CRISPIM, C. A.; STULBACH, T. E. Aplicação da Escala de Conhecimento Nutricional em atletas profissionais 
Avaliação da influência de características sensoriais e do conhecimento nutricional na aceitação do chá-mate

Barboza, H. C.; Cazal, M. M.

e amadores de atletismo. Revista Brasileira de Medicina do Esporte, v. 14, n. 3, p. 205-208, 2008. http://dx.doi.org/10.1590/ S1517-86922008000300009.

PRATES, R. E.; SILVA, A. C. P. Avaliação do conhecimento nutricional e de hábitos alimentares de pacientes com doenças crônicas não transmissíveis em hospital particular no sul do Brasil. Revista da Associação Brasileira de Nutrição, v. 5, n. 1, p. 21-27, 2013.

PRECI, D.; CHICOSKI, A. J.; VALDUGA, A. T.; OLIVEIRA, D.; VALDUGA, E.; TREICHEL, H.; TONIAZZO, G.; CANSIAN, R. L. Desenvolvimento de iogurte light com extrato de erva mate (Ilex paraguariensis ST. HIL) e adição de probióticos. Alimentos e Nutrição, v. 22, n. 1, p. 27-38, 2011.

RIBEIRO, M. M.; DELLA LUCIA, S. M.; BARBOSA, P. B. F.; GALVÃO, H. L.; MINIM, V. P. R. Influência da embalagem na aceitação de diferentes marcas comerciais de cerveja tipo Pilsen. Ciência e Tecnologia de Alimentos, v. 28, n. 2, p. 395-399, 2008. http:// dx.doi.org/10.1590/S0101-20612008000200019.

ROCHA, F. A. Percepção dos consumidores de alimentos acerca dos conceitos de alimentos light e diet e das informações contidas nos rótulos. 2008. 17 f. Monografia (Trabalho de Conclusão em Higiene e Inspeção de Produtos de Origem Animal)Universidade Castelo Branco, Belo Horizonte, 2008.

SAIGG, N. L.; SILVA, M. C. Efeitos da utilização do chá verde na saúde humana. Universitas: Ciências da Saúde, v. 7, n. 1, p. 69-89, 2009.
SILVA, M. Z. T. Influência da rotulagem na rotulagem nutricional sobre o consumidor. 2003. 67 f. Dissertação (Mestrado em Nutrição)-Universidade Federal de Pernambuco, Recife, 2003.

SOUZA, S. M. F. C.; LIMA, K. C.; MIRANDA, H. F.; CAVALCANTI, F. I. D. Utilização da informação nutricional de rótulos por consumidores de Natal, Brasil. Revista Panamericana de Salud Pública, v. 29, n. 5, p. 337-343, 2011. PMid:21709938.

StEFANO, N. M.; CASAROTTO FILHO, N. Percepção dos consumidores: atributos considerados importantes nas embalagens. Revista Produção Online, v. 12, n. 3, p. 657-681, 2012. http:// dx.doi.org/10.14488/1676-1901.v12i3.962.

TEIXEIRA, L. V. Análise sensorial na indústria de alimentos. Revista do Instituto de Laticínios Cândido Tostes, v. 64, n. 366, p. 1-14, 2009.

WANG, S. H.; CABRAL, L. C.; ARAUJO, F. B.; MAIA, L. H. Características sensoriais de leites de soja reconstituídos. Pesquisa Agropecuária Brasileira, v. 34, n. 3, p. 467-472, 1999. http://dx.doi.org/10.1590/S0100-204X1999000300019.

ZANCHETT, C. S.; MIGNONI, M. L.; BARRO, N. P. R.; ROSA, C. $D$. Desenvolvimento de chocolate branco com extrato de ervamate. Brazilian Journal of Food Technology, v. 19, p. 2-8, 2016. http://dx.doi.org/10.1590/1981-6723.7315. 